(2) Open Access Full Text Article

\title{
Spectral-domain optical coherence tomography imaging of macula-off rhegmatogenous retinal detachment
}

\author{
This article was published in the following Dove Press journal: \\ Clinical Ophthalmology \\ 18 March 2014 \\ Number of times this article has been viewed
}

\author{
Jayanth Sridhar \\ Harry W Flynn Jr \\ Department of Ophthalmology, \\ Bascom Palmer Eye Institute, \\ Miami, FL, USA
}

Correspondence: Jayanth Sridhar Bascom Palmer Eye Institute, 900 NW 17th Street Miami, FL 33।36, USA

$\mathrm{Tel}+\mathrm{I} 305326$ 6I I8

Email jsridhar@med.miami.edu

\begin{abstract}
The timing of repair and the utility of spectral-domain optical coherence tomography (SD-OCT) for macula-off retinal detachment remains a controversial issue. Four patients who presented with macula-off retinal detachment underwent repair at various time points after symptom onset. Postoperative SD-OCT of the macula demonstrated correlation with final visual acuity.
\end{abstract}

Keywords: SD-OCT, vitrectomy, scleral buckle

\section{Introduction}

Despite extensive study, timing of repair for macula-off rhegmatogenous retinal detachments remains controversial. It has been suggested that visual recovery is correlated with the height of a bullous macula-off detachment. ${ }^{1}$ It has also been reported that shorter duration of macular detachment is associated with better visual acuity outcomes. ${ }^{2}$ Recent studies have reported the use of spectral-domain optical coherence tomography (SD-OCT) in the evaluation and follow-up of patients with retinal detachment and retinoschisis. ${ }^{3,4}$ In the current study, four patients with macula-off retinal detachment are reported that underwent surgical repair at various time points after symptom onset with preoperative and postoperative clinical and anatomic correlation using SD-OCT.

\section{Case report Case I}

A 70-year-old Latin American woman presented for evaluation of decreased vision in the right eye for 3 days. Past ocular history was significant for bilateral uncomplicated cataract extraction with intraocular lens placement 4 months prior.

Ophthalmic examination revealed best-corrected visual acuity of 1/200 in the right eye and 20/20 in the left. A relative afferent pupillary defect was noted in the right eye. Intraocular pressure was $14 \mathrm{mmHg}$ in both eyes. The posterior segment examination of the right eye demonstrated a bullous rhegmatogenous retinal detachment caused by a superotemporal horseshoe tear (Figure 1A). SD-OCT revealed marked elevation of the retina extending through the macula to the optic nerve (Figure 1B).

The patient underwent a scleral buckling procedure with cryotherapy 6 days after initial examination. The following day, $0.3 \mathrm{~cm}^{3}$ of $100 \% \mathrm{C}_{3} \mathrm{~F}_{8}$ (octafluoropropane) gas was placed in the right eye because of persistent subretinal fluid. Postoperatively, the patient's retina remained attached, and vision slowly improved. Six months after 

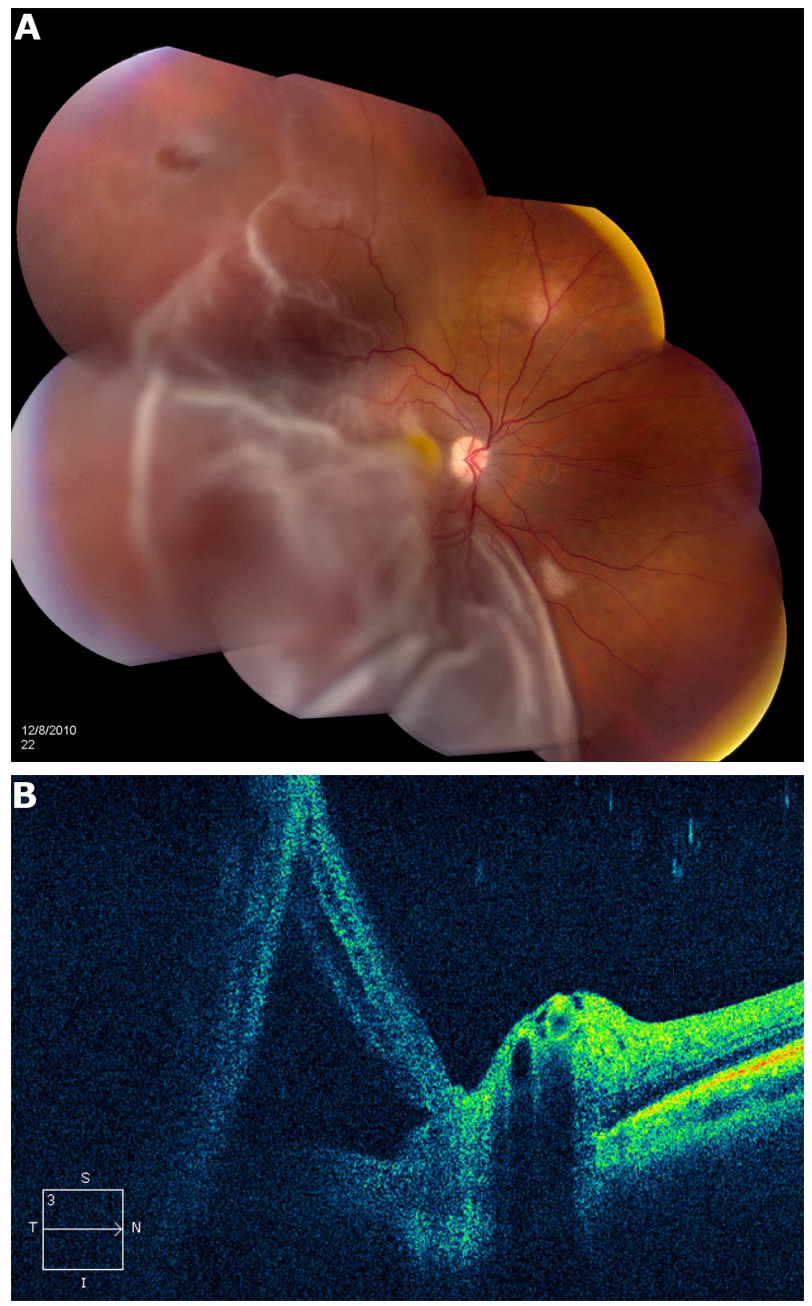

Figure I Preoperative examination, Case I. (A) Right eye posterior segment photograph demonstrating bullous retinal detachment extending to optic nerve from superotemporal horseshoe flap tear. (B) Right eye SD-OCT demonstrating bullous retinal detachment, with subretinal fluid extending to optic nerve (Cirrus ${ }^{\mathrm{TM}}$ HD-OCT; Carl Zeiss Meditec, Inc., Dublin, CA, USA).

Abbreviation: SD-OCT, spectral-domain optical coherence tomography.

successful retinal detachment surgery, the patient's bestcorrected visual acuity in the right eye was 20/60 (Figure 2A). SD-OCT revealed mild foveal atrophy in the outer nuclear layer, with mild disruption of the inner segment ellipsoid layer (Figure 2B).

\section{Case 2}

A 57-year-old Latin American woman presented for evaluation of decreased vision in the right eye for 2 days. Past ocular history was significant for bilateral uncomplicated cataract extraction with intraocular lens placement 5 months prior and a retinal tear in the right eye, status post-laser retinopexy.

Ophthalmic examination revealed best-corrected visual acuity of 20/70 in the right eye and 20/20 in the left eye. No relative afferent pupillary defect was noted. Intraocular
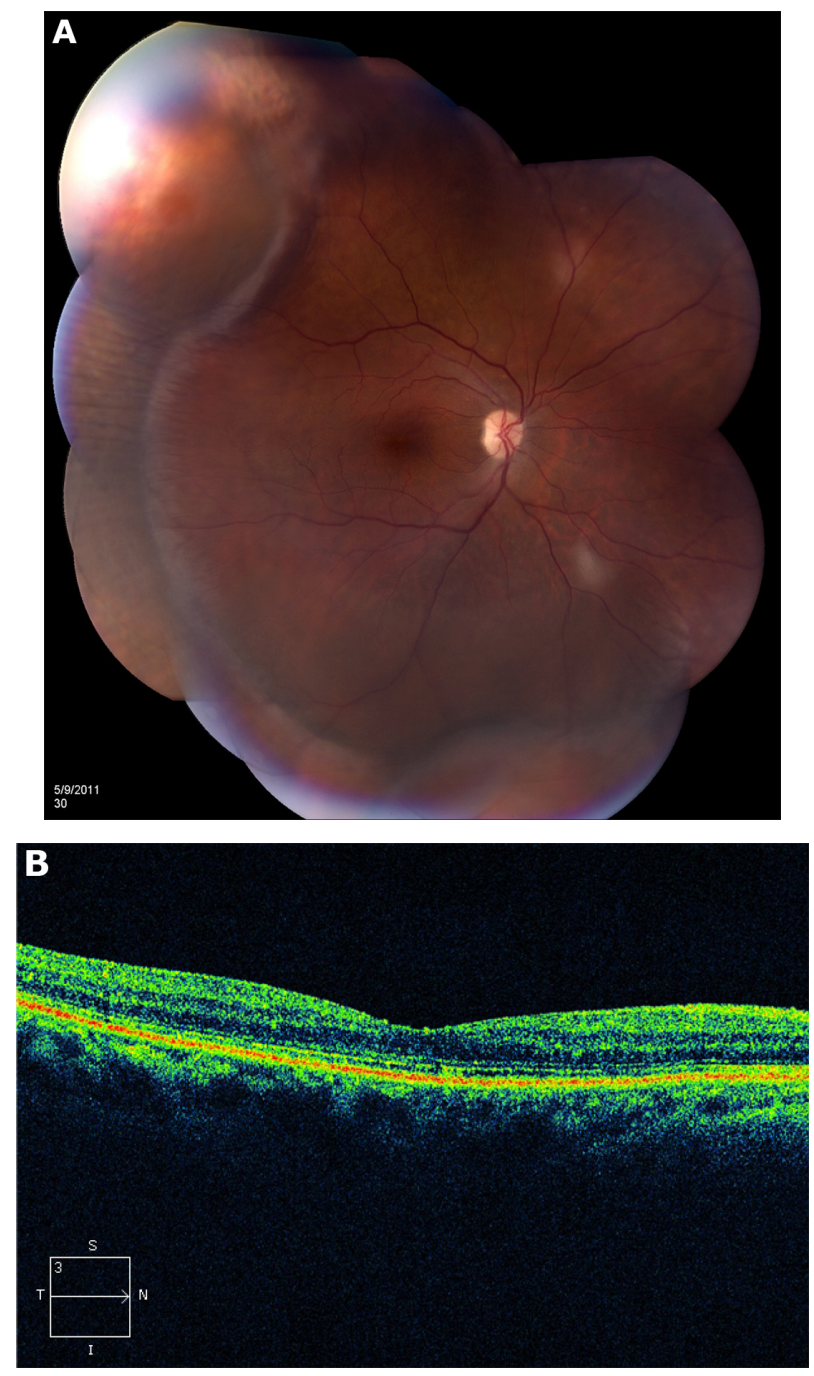

Figure 2 Postoperative examination, Case I. (A) Right eye posterior segment examination with visible scleral buckle and reattached macula. (B) Right eye SDOCT demonstrating mild disruption of the inner segment ellipsoid layer (Cirrus ${ }^{\mathrm{TM}}$ HD-OCT; Carl Zeiss Meditec, inc., Dublin, CA, USA).

Abbreviation: SD-OCT, spectral-domain optical coherence tomography.

pressure was $15 \mathrm{mmHg}$ in the right eye and $18 \mathrm{mmHg}$ in the left eye. The posterior segment examination of the right eye revealed a shallow rhegmatogenous retinal detachment caused by two temporal horseshoe tears (Figure 3A). SD-OCT demonstrated temporal subretinal fluid extending under the fovea (Figure 3B).

The patient underwent pars plana vitrectomy with air-fluid exchange, endolaser, and placement of $\mathrm{C}_{3} \mathrm{~F}_{8}$ (octafluoropropane) gas in the right eye 1 day after presentation. Postoperatively, the patient's retina remained attached, and vision slowly improved. One year after surgery, the patient's best-corrected visual acuity in the right eye was 20/20 (Figure 4A). SD-OCT revealed intact foveal contour with normal-appearing inner segment ellipsoid layer (Figure 4B). 

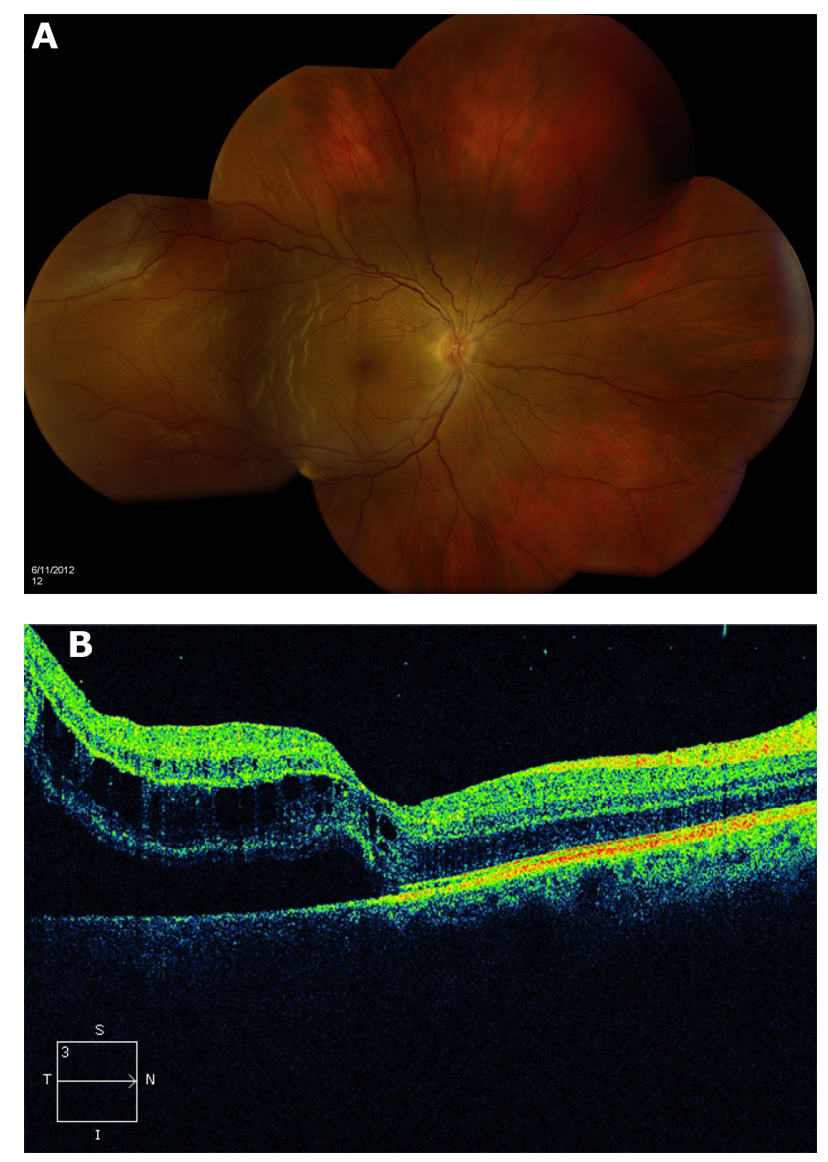

Figure 3 Preoperative examination, Case 2. (A) Right eye posterior segment examination demonstrating a temporal retinal detachment, with subretinal fluid extending into the fovea. (B) Right eye SD-OCT demonstrating retinal detachment with subretinal fluid extending beneath the fovea (Cirrus ${ }^{\mathrm{TM}}$ HD-OCT; Carl Zeiss Meditec, Inc., Dublin, CA, USA).

Abbreviation: SD-OCT, spectral-domain optical coherence tomography.

\section{Case 3}

A 49-year-old Caucasian woman presented with history of floaters in the right eye for 3 weeks and decreased vision for 1 day. Past ocular history was significant for bilateral LASIK (Laser-Assisted in situ Keratomileusis) for myopia 10 years prior.

Ophthalmic examination revealed best-corrected visual acuity of 20/200 in the right eye and 20/20 in the left eye. No relative afferent pupillary defect was noted. Intraocular pressure was $16 \mathrm{mmHg}$ in both eyes. The posterior segment examination demonstrated a bullous rhegmatogenous retinal detachment caused by a superior horseshoe tear (Figure 5A). SD-OCT showed extensive subretinal fluid, with bullous elevation of the fovea (Figure 5B).

The patient underwent a successful scleral buckling procedure with placement of $0.5 \mathrm{~cm}^{3}$ of $100 \% \mathrm{SF}_{6}$ (sulfur hexafluoride) gas. One month after surgery the patient's bestcorrected visual acuity in the right eye was 20/40 (Figure 6A).
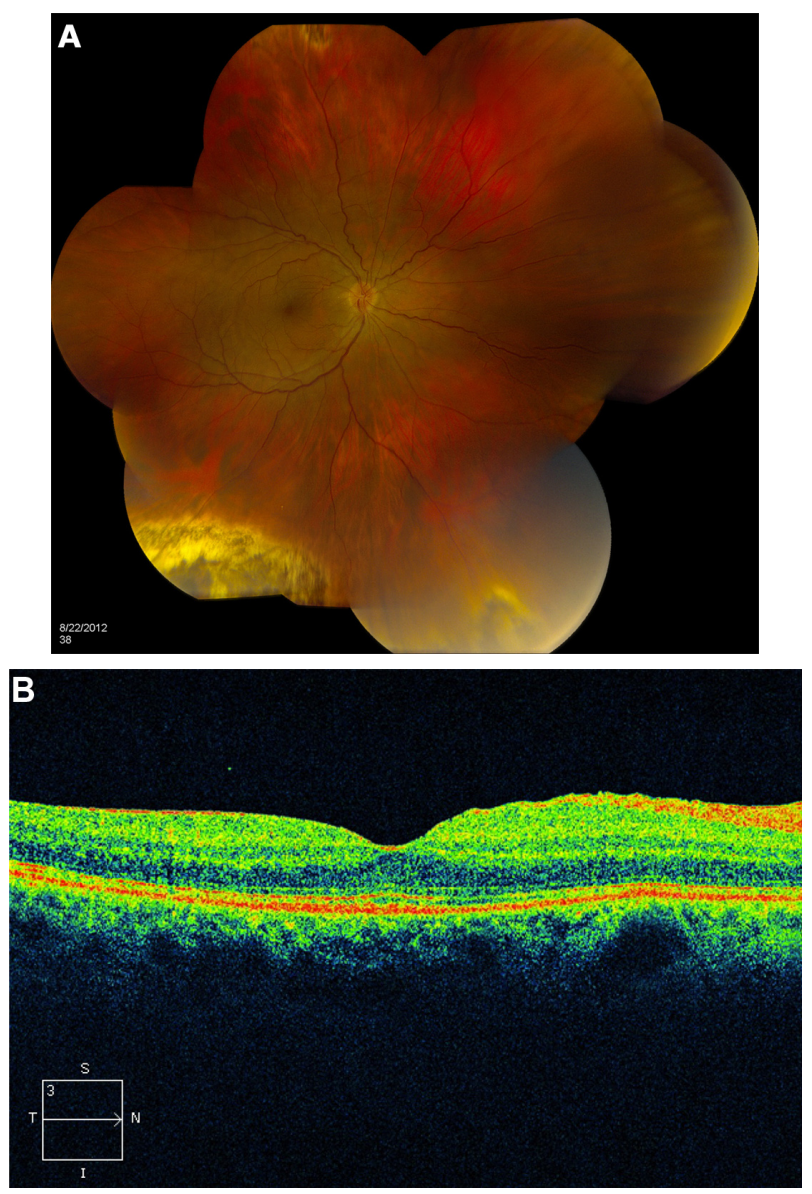

Figure 4 Postoperative examination, Case 2. (A) Right eye posterior segment examination revealing attached retina with good foveal reflex. (B) Right eye SDOCT demonstrating intact foveal contour and inner segment ellipsoid layer with nasal epiretinal membrane (Cirrus ${ }^{\mathrm{TM}}$ HD-OCT; Carl Zeiss Meditec, inc., Dublic, CA, USA).

Abbreviation: SD-OCT, spectral-domain optical coherence tomography.

SD-OCT revealed intact foveal contour with normalappearing inner segment ellipsoid layer (Figure 6B).

\section{Case 4}

A 50-year-old Caucasian woman presented with history of gradual decrease in vision in the right eye over several months. Past ocular history was significant for bilateral LASIK for myopia 1 year prior.

Ophthalmic examination revealed best-corrected visual acuity of 20/100 in the right eye and 20/15 in the left eye. No relative afferent pupillary defect was noted. Intraocular pressure was $10 \mathrm{mmHg}$ in both eyes. The posterior segment examination demonstrated a rhegmatogenous retinal detachment caused by inferior retinal holes (Figure 7A). SD-OCT revealed shallow subretinal fluid extending under the fovea (Figure 7B).

The patient underwent a successful scleral buckling procedure with cryotherapy. At 3-month follow-up the patient's 

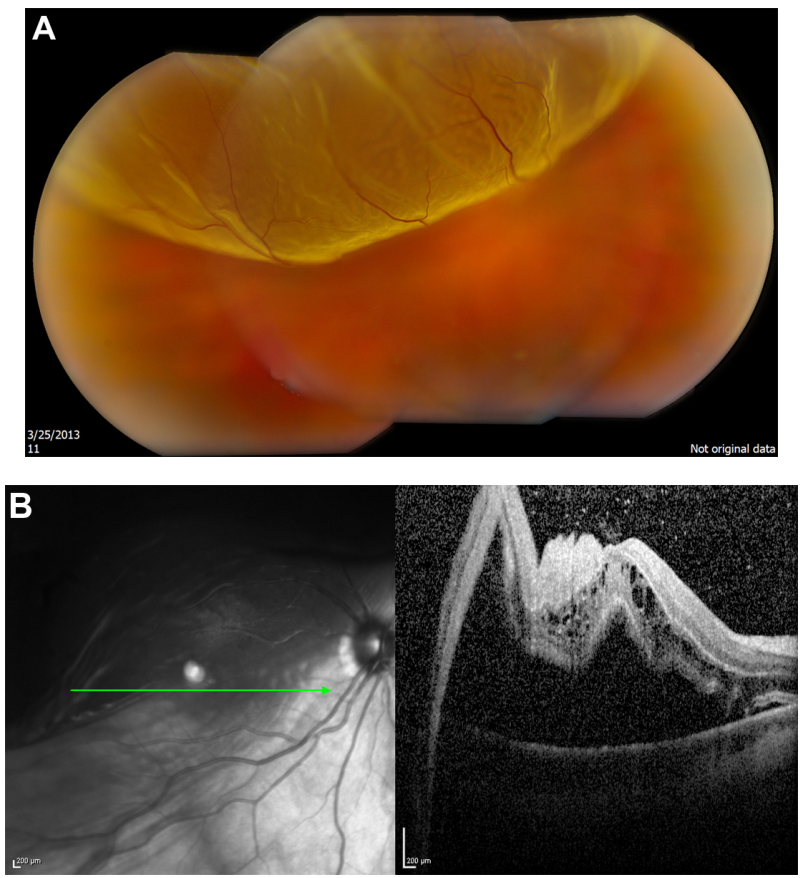

Figure 5 Preoperative examination, Case 3. (A) Right eye posterior segment examination demonstrating a superior bullous retinal detachment with subretinal fluid extending into the fovea. (B) Right eye SD-OCT demonstrating subretinal fluid, with bullous elevation of the fovea (Spectralis HRA + OCT; Heidelberg Engineering, Inc., Heidelberg, Germany).

Abbreviation: SD-OCT, spectral-domain optical coherence tomography.
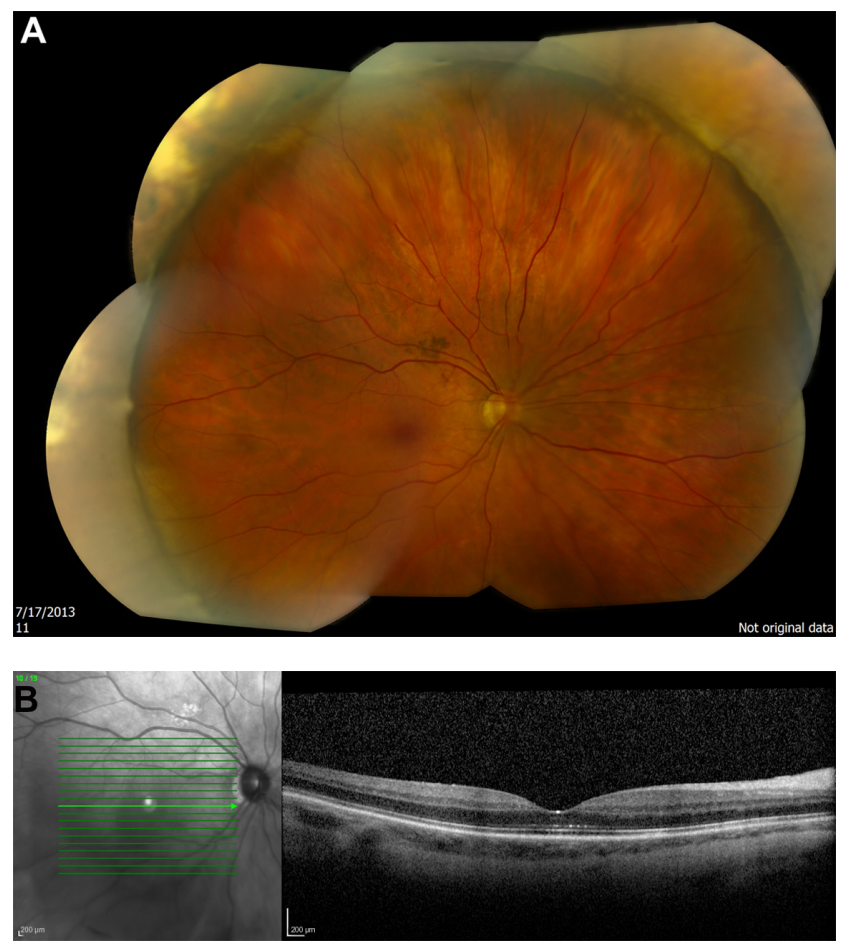

Figure 6 Postoperative examination, Case 3. (A) Right eye posterior segment examination revealing attached retina with good foveal reflex. (B) Right eye SD-OCT demonstrating intact foveal contour and inner segment ellipsoid layer (Spectralis HRA + OCT; Heidelberg Engineering, Inc., Heidelberg, Germany).

Abbreviation: SD-OCT, spectral-domain optical coherence tomography.
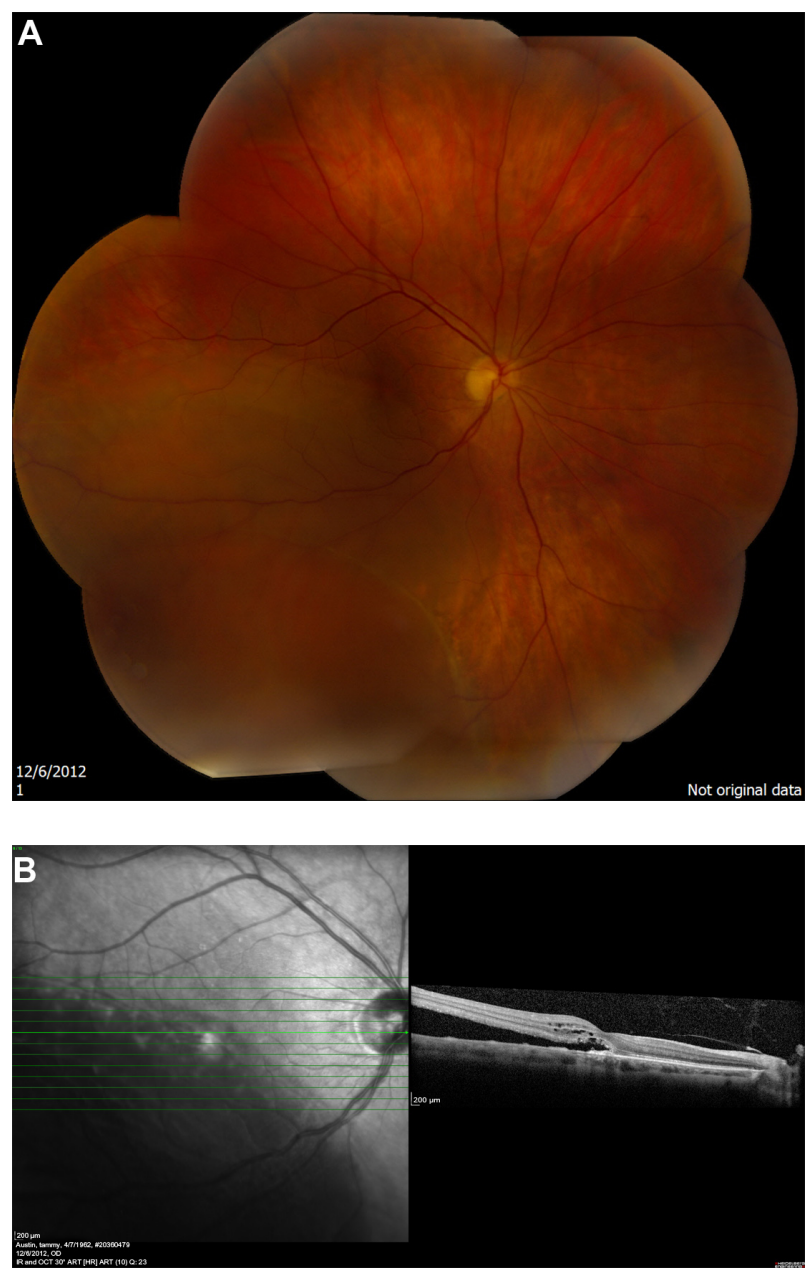

Figure 7 Preoperative examination, Case 4. (A) Right eye posterior segment examination demonstrating an inferior retinal detachment, with subretinal fluid extending into the fovea. (B) Right eye SD-OCT demonstrating subretinal fluid extending through the fovea (Spectralis HRA + OCT; Heidelberg Engineering, Inc., Heidelberg, Germany).

Abbreviation: SD-OCT, spectral-domain optical coherence tomography.

best-corrected visual acuity in the right eye was $20 / 200$ (Figure 8A). SD-OCT revealed intact foveal contour with disruption of the inner segment ellipsoid layer (Figure 8B).

\section{Discussion}

With an annual incidence of 12 per 100,000 individuals, rhegmatogenous retinal detachment remains an important cause of visual morbidity, especially in macula-off cases. ${ }^{5}$ Risk factors for retinal detachment include prior retinal tears, myopia, and cataract surgery. ${ }^{6}$ Options for surgical repair include traditional scleral buckling, pars plana vitrectomy, pneumatic retinopexy, and combination techniques, with regional variation in the choice between these procedures. ${ }^{7}$ The strongest prognostic indicator of postoperative visual outcome is preoperative visual acuity. ${ }^{8}$ 

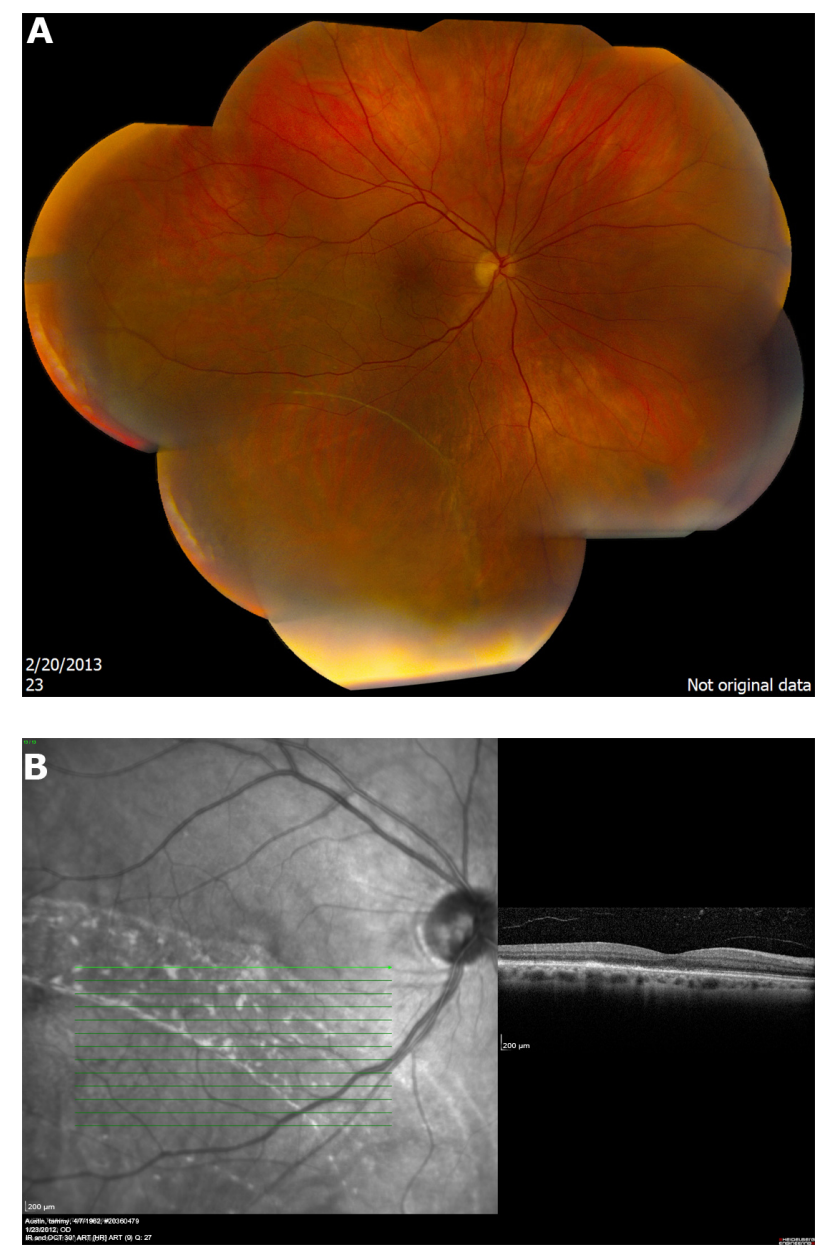

Figure 8 Postoperative examination, Case 4. (A) Right eye posterior segment examination revealing attached retina with pigment demarcation line inferiorly. (B) Right eye SD-OCT demonstrating intact foveal contour, resolution of cystoid macular edema, and moderate disruption of the inner segment ellipsoid layer (Spectralis HRA + OCT; Heidelberg Engineering, Inc., Heidelberg, Germany). Abbreviation: SD-OCT, spectral-domain optical coherence tomography.

A controversial issue surrounding surgical repair of maculaoff rhegmatogenous retinal detachments is the timing of repair. The first three patients described in the cases underwent repair 9, 3, and 1 day(s) after symptom onset, respectively. It has been proposed that rapid repair resulted in better visual acuity outcomes. One case series demonstrated that macular detachment of less than 10 days was associated with better final visual acuity. ${ }^{2}$ More recently, one group proposed that surgical repair within 6 days of symptoms of macular detachment resulted in similar anatomic outcomes but better visual acuity outcomes as compared with repair beyond 6 days. ${ }^{9}$ However, in another study, there was no statistical difference in visual recovery in patients operated within 1-2 days, 3-4 days, or 5-7 days after macular involvement. ${ }^{10}$

It has been recognized that successful anatomic reattachment does not always predict visual improvement in macula-off cases. Although the retina was successfully attached in all four patients, the final best-corrected visual acuity in the fourth patient was 20/200 as compared with 20/60 in the first patient, 20/20 in the second patient, and 20/40 in the third patient. On postoperative SD-OCT, the first and fourth patients had outer nuclear atrophy and inner segment ellipsoid layer disruption, while the other patients did not. Despite reattachment, there are microstructural changes within the photoreceptor layer, specifically disruption of the inner segment ellipsoid layer, in SD-OCT. ${ }^{11}$ One study demonstrated that the status of the inner segment ellipsoid layer as well as the outer nuclear layer thickness are significantly correlated with final visual acuity outcomes after retinal detachment repair. ${ }^{12}$ It has been shown that increased preoperative foveal thickness and outer retinal corrugation are associated with worse visual prognosis, perhaps due to ellipsoid layer disruption. ${ }^{3,13}$ However, the majority of cases, including in these studies, were not bullous retinal detachments.

These four patients with macula-off retinal detachments demonstrate anatomical findings that correlate with final visual acuity outcome using SD-OCT. This technology is usually applied to conditions without bullous retinal detachment, but this case series shows that the technology can also be applied in this condition. While the exact timing of repair for macula-off detachments remains controversial, SD-OCT and other newer imaging modalities may be helpful as prognostic tools both before and after retinal detachment surgery. Further study, such as a larger retrospective series with masked reading of postoperative SD-OCT images, is recommended.

\section{Disclosure}

This research was funded in part by an unrestricted grant from Research to Prevent Blindness Inc., New York, NY, USA and the National Institutes of Health NEI Center Grant P30 EY014801. Dr Flynn is a consultant for Santen and Vindico. The authors have no proprietary interests in the materials described in this article.

\section{References}

1. Ross W, Lavina A, Russell M, Maberley D. The correlation between height of macular detachment and visual outcome in macula-off retinal detachments of $\leq 7$ days' duration. Ophthalmology. 2005;112(7): 1213-1217.

2. Hassan TS, Sarrafizadeh R, Ruby AJ, Garretson BR, Kuczynski B, Williams GA. The effect of duration of macular detachment on results after scleral buckle repair of primary, macula-off retinal detachments. Ophthalmology. 2002;109(1):146-152. 
3. Cho M, Witmer MT, Favarone G, Chan RP, D'Amico DJ, Kiss S. Optical coherence tomography predicts visual outcome in maculainvolving rhegmatogenous retinal detachment. Clin Ophthalmol. 2012; 6:91-96.

4. Stehouwer M, Tan SH, van Leeuwen TG, Verbraak FD. Senile retinoschisis versus retinal detachment, the additional value of peripheral retinal OCT scans (SL SCAN-1, Topcon). Acta Ophthalmol. Epub June 15, 2013.

5. Sodhi A, Leung LS, Do DV, Gower EW, Schein OD, Handa JT. Recent trends in the management of rhegmatogenous retinal detachment. Surv Ophthalmol. 2008;53(1):50-67.

6. Olsen T, Jeppesen P. The incidence of retinal detachment after cataract surgery. Open Ophthalmol J. 2012;6:79-82.

7. Schwartz SG, Flynn HW Jr. Primary retinal detachment: scleral buckle or pars plana vitrectomy? Curr Opin Ophthalmol. 2006;17(3):245-250.

8. Burton TC, Lambert RW Jr. A predictive model for visual recovery following retinal detachment surgery. Ophthalmology. 1978;85(6):619-625.
9. Kim JD, Pham HH, Lai MM, Josephson JW, Minarcik JR, Von Fricken M. Effect of symptom duration on outcomes following vitrectomy repair of primary macula-off retinal detachments. Retina. 2013;33(9):1931-1937.

10. Ross WH, Kozy DW. Visual recovery in macula-off rhegmatogenous retinal detachments. Ophthalmology. 1998;105(11):2149-2153.

11. Delolme MP, Dugas B, Nicot F, Muselier A, Bron AM, CreuzotGarcher C. Anatomical and functional macular changes after rhegmatogenous retinal detachment with macula off. Am J Ophthalmol. 2012;153(1):128-136.

12. Gharbiya M, Grandinetti F, Scavella V, et al. Correlation between spectral-domain optical coherence tomography findings and visual outcome after primary rhegmatogenous retinal detachment repair. Retina. 2012;32(1):43-53.

13. Ricker LJ, Noordzij LJ, Goezinne F, et al. Persistent subfoveal fluid and increased preoperative foveal thickness impairs visual outcome after macula-off retinal detachment repair. Retina. 2011;31(8):1505-1512.
Clinical Ophthalmology

\section{Publish your work in this journal}

Clinical Ophthalmology is an international, peer-reviewed journal covering all subspecialties within ophthalmology. Key topics include: Optometry; Visual science; Pharmacology and drug therapy in eye diseases; Basic Sciences; Primary and Secondary eye care; Patient Safety and Quality of Care Improvements. This journal is indexed on

\section{Dovepress}

PubMed Central and CAS, and is the official journal of The Society of Clinical Ophthalmology (SCO). The manuscript management system is completely online and includes a very quick and fair peer-review system, which is all easy to use. Visit http://www.dovepress.com/ testimonials.php to read real quotes from published authors. 